\title{
A Design Method of Saturation Test Image Based on CIEDE2000
}

\author{
Yang Yang, ${ }^{1,2}$ Jun Ming, ${ }^{2}$ and Nenghai Yu' ${ }^{1}$ \\ ${ }^{1}$ MOE-Microsoft Key Lab of Multimedia Computing \& Communication, \\ University of Science and Technology of China, Hefei 230027, China \\ ${ }^{2}$ Key IC¿SP Lab of Ministry of Education, Anhui University, Hefei 230039, China \\ Correspondence should be addressed to Yang Yang, skyyang@mail.ustc.edu.cn \\ Received 11 May 2012; Accepted 25 June 2012 \\ Academic Editor: Steven C. H. Hoi
}

Copyright ( $) 2012$ Yang Yang et al. This is an open access article distributed under the Creative Commons Attribution License, which permits unrestricted use, distribution, and reproduction in any medium, provided the original work is properly cited.

In order to generate color test image consistent with human perception in aspect of saturation, lightness, and hue of image, we propose a saturation test image design method based on CIEDE2000 color difference formula. This method exploits the subjective saturation parameter $C^{\prime}$ of CIEDE2000 to get a series of test images with different saturation but same lightness and hue. It is found experimentally that the vision perception has linear relationship with the saturation parameter $C^{\prime}$. This kind of saturation test image has various applications, such as in the checking of color masking effect in visual experiments and the testing of the visual effects of image similarity component.

\section{Introduction}

With the rapid development of television technology, communication technology, and computer technology, image quality assessment field has attracted more and more researchers [1-5]. Huge amount of effects were put to seek for an objective quality assessment metric which cannot only be calculated simply, but also accurately reflect subjective quality of human perception [1-3]. The key focus is to reduce deviation between the subjective and objective quality assessment results. The image test database is essential for comparing the deviation between the subjective and objective quality assessment results [6-8]. At present, some research institutes have provided some image test databases such as LIVE database provided by images and video engineering laboratory of the University of Texas at Austin and so on. But in some cases, we need some special designed test images to study the exact visual effect of individual parameter such as saturation, lightness, and hue.

To study the masking effect of color image in visual experiments, we need a series of special designed test images in which one of color features (hue, lightness, and saturation) is varied and the other two are kept unchanged. For example, with a series of images, named saturation test images, having the same hue and lightness but different saturation, we can add same noise signal on all these saturation test images and test observation influence of different saturation test images to noise signal. In addition, this kind of saturation test images has the same structure so that it can be used to test the visual effects of image similarity components [9].

CIEDE2000 color difference formula is established from the nonlinear perception subjective experiment of HVS model, in which lightness, hue, and saturation parameters are consistent with subjective visual perceptional [10-12]. In order to further inspect the saturation, hue, or lightness subjective feeling in CIEDE2000, it is valuable to find a test image design method directly based on CIEDE2000 color difference formula.

In this paper, we propose a saturation test image design method based on the subjective saturation parameters $C^{\prime}$ of CIEDE2000 color difference formula. This kind of saturation test images with different saturation but same lightness and hue $\left(\Delta L^{\prime}=0, \Delta H^{\prime}=0\right)$. Our experiment shows that nice consistency is found between saturation test images and subjective visual perceptional. The vision perception changes linear when the saturation parameters $C^{\prime}$ of test images increased step by step. This kind of saturation test signals has various applications, such as in the checking of color masking effect in visual experiments and the testing of the visual effects of image similarity component. The idea involved in 


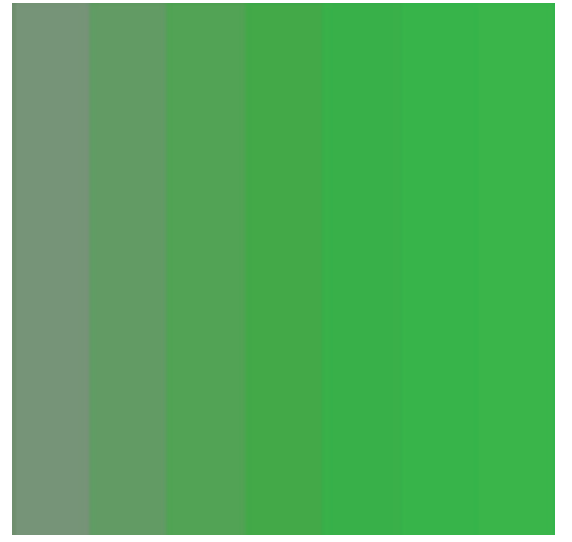

FIGURE 1: The equal difference of saturation test ladder images.

this method can be also exploited to design other test images such as hue or lightness test images.

\section{The Design Condition of Saturation Test Image Based on CIEDE2000}

The proposed saturation test image design method based on the CIEDE2000 color difference formula enables us to get test images having difference saturation but similar lightness and hue, in comparison with reference image. Thus, from CIEDE2000 color difference formula, we can get $\Delta L^{\prime}=0$ and $\Delta H^{\prime}=0$, where $\Delta L^{\prime}$ and $\Delta H^{\prime}$, respectively, represent the lightness and hue difference. This is the design condition for designing the saturation test image.

In CIEDE2000 color difference formula, $\Delta L^{\prime}=L_{2}^{\prime}-L_{1}^{\prime}$ as $L_{1}^{\prime}, L_{2}^{\prime}$ are the lightness of reference image and test image, respectively. The condition $\Delta L^{\prime}=0$ implies $L_{2}^{\prime}=L_{1}^{\prime}$.

In CIEDE2000, there is,

$$
\begin{gathered}
L_{i}^{*}=L_{i}^{\prime}, \\
L_{i}^{*}=116 f\left(\frac{Y_{i}}{Y_{0}}\right)-16,
\end{gathered}
$$

thus, we can get $f\left(Y_{1} / Y_{0}\right)=f\left(Y_{2} / Y_{0}\right)$. Here

$$
f(I)= \begin{cases}I^{1 / 3}, & \text { for } I>0.008856 \\ 7.787 I+\frac{16}{116}, & \text { otherwise }\end{cases}
$$

where $I=X_{i} / X_{0}, Y_{i} / Y_{0}$, and $Z_{i} / Z_{0}$. From these calculations, we can get the result $Y_{1}=Y_{2}$ from $\Delta L^{\prime}=0$.

In CIEDE2000 color difference formula, the hue difference parameter $\Delta H^{\prime}=2 \sqrt{C_{1}^{\prime} C_{2}^{\prime}} \cdot \sin \left(\Delta h^{\prime} / 2\right)$, where $C_{1}^{\prime}$, $C_{2}^{\prime}$ are the saturation of reference image and test image, respectively. The difference of hue angle is $\Delta h^{\prime}=h_{2}^{\prime}-$ $h_{1}^{\prime}=\tan ^{-1}\left(b_{2}^{\prime}, a_{2}^{\prime}\right)-\tan ^{-1}\left(b_{1}^{\prime}, a_{1}^{\prime}\right)$. Thus, $\Delta H^{\prime}=0$ implies $\tan ^{-1}\left(b_{2}^{\prime}, a_{2}^{\prime}\right)=\tan ^{-1}\left(b_{1}^{\prime}, a_{1}^{\prime}\right)$. According to the CIEDE2000, there is $a_{i}^{\prime}=a_{i}^{*}(1+G)$. Thus, we can get

$$
\tan ^{-1}\left(b_{2}^{*}, a_{2}^{*}(1+G)\right)=\tan ^{-1}\left(b_{1}^{*}, a_{1}^{*}(1+G)\right) .
$$

Simplifying it, we can reach the equations

$$
\begin{gathered}
a_{1}^{*} b_{2}^{*}=b_{1}^{*} a_{2}^{*} \quad \text { if }\left(a_{1}^{*} \neq 0\right), \\
a_{2}^{*}=0, \quad b_{2}^{*}=b_{1}^{*} \quad \text { if }\left(a_{1}^{*}=0\right) .
\end{gathered}
$$

Thus, from $\Delta L^{\prime}=0$ and $\Delta H^{\prime}=0$, we can get the design conditions of saturation test image as

$$
Y_{2}=Y_{1}, \quad \begin{array}{cc}
a_{1}^{*} b_{2}^{*}=b_{1}^{*} a_{2}^{*} & \text { if }\left(a_{1}^{*} \neq 0\right), \\
a_{2}^{*}=0, \quad b_{2}^{*}=b_{1}^{*} & \text { if }\left(a_{1}^{*}=0\right) .
\end{array}
$$

\section{The Procedure of Saturation Test Image Design Method}

The calculation of the CIEDE2000 color difference formula is complicated as it involved the conversion of four CIE (CIEDE2000, CIELAB, CIE XYZ, and CIE RGB). It involves to the transcendental equation solving when use $\Delta E$ parameter and (5) as the conditions to design test image. Here we use $C_{2}^{\prime}=K * C_{1}^{\prime}$ as the initial conditions to design the saturation test image to simplify the calculation processing, where $C_{1}^{\prime}, C_{2}^{\prime}$ are the saturation of reference image and test image, respectively. The detailed saturation test image design steps are listed as follows.

(1) $R_{1}, G_{1}$, and $B_{1}$ are the given parameters for reference image, and we will calculate $R_{2}, G_{2}$, and $B_{2}$ for the saturation test image.

(2) We choose the parameters $X_{0}, Y_{0}$, and $Z_{0}$ of standard light source as the same way in [13].

(3) The $X_{1}, Y_{1}$, and $Z_{1}$ of reference image are given by

$$
\left(\begin{array}{c}
X_{1} \\
Y_{1} \\
Z_{1}
\end{array}\right)=A\left(\begin{array}{l}
R_{1} \\
G_{1} \\
B_{1}
\end{array}\right)
$$

The matrix $A$ is chosen also as [13].

(4) According to the CIEDE2000 $f\left(X_{1} / X_{0}\right), f\left(Y_{1} / Y_{0}\right)$, and $f\left(Z_{1} / Z_{0}\right)$ are given by

$$
f(I)= \begin{cases}I^{1 / 3}, & \text { if } I>0.008856, \\ 7.787 I+\frac{16}{116}, & \text { otherwise, }\end{cases}
$$

where $I=X_{i} / X_{0}, Y_{i} / Y_{0}, Z_{i} / Z_{0}$.

(5) According to the CIEDE2000, $a_{1}^{*}, a_{2}^{*}$ are given by $a_{1}^{*}=500\left[f\left(X_{1} / X_{0}\right)-f\left(Y_{1} / Y_{0}\right)\right]$, and $b_{1}^{*}=$ $200\left[f\left(Y_{1} / Y_{0}\right)-f\left(Z_{1} / Z_{0}\right)\right]$.

(6) According to the CIEDE2000, there is $C_{i}^{\prime}=$ $\sqrt{\left(a_{i}^{\prime}\right)^{2}+\left(b_{i}^{\prime}\right)^{2}}, a_{i}^{\prime}=(1+G) a_{i}^{*}$, and $b_{i}^{\prime}=b_{i}^{*}$. We use $C_{2}^{\prime}=K * C_{1}^{\prime}$ as the initial condition to design saturation of test image, where $K$ is a real number chosen according to the required saturation of designing test image. Then we can get $(1+G)^{2}\left(a_{2}^{*}\right)^{2}+\left(b_{2}^{*}\right)^{2}=$ $K^{2}\left((1+G)^{2}\left(a_{1}^{*}\right)^{2}+\left(b_{1}^{*}\right)^{2}\right)$. Combine it with (4), we can get

$$
\begin{gathered}
\left|a_{2}^{*}\right|=\left|K * a_{1}^{*}\right|, \quad b_{2}^{*}=\frac{b_{1}^{*}}{a_{1}^{*}} a_{2}^{*} \quad \text { if }\left(a_{1}^{*} \neq 0\right), \\
a_{2}^{*}=0, \quad b_{2}^{*}=b_{1}^{*} \quad \text { if }\left(a_{1}^{*}=0\right) .
\end{gathered}
$$


TABLE 1: The corresponding experimental calculating parameter of Figure 1.

\begin{tabular}{lcccccccc}
\hline$K_{n}$ & $R$ & $G$ & $B$ & $X$ & $Y$ & $Z$ & $a^{*}$ \\
\hline 1 & 120 & 150 & 120 & 48.5176 & 55.1176 & 52.5882 & -10.4799 \\
2 & 100 & 159 & 101 & 44.7985 & 55.1176 & 45.8335 & -20.9597 & 14.0602 \\
3 & 81 & 168 & 84 & 41.2744 & 55.1176 & 39.6834 & -31.4396 \\
4 & 62 & 176 & 68 & 37.9402 & 55.1176 & 34.1097 & -41.9194 & 21.0903 \\
5 & 45 & 184 & 54 & 34.7905 & 55.1176 & 29.0839 & -52.3993 \\
6 & 28 & 191 & 41 & 31.8202 & 55.1176 & 24.5778 & -62.8791 & 42.1804 \\
7 & 12 & 198 & 30 & 29.0239 & 55.1176 & 20.5629 & -73.3590 & 49.2108 \\
\hline
\end{tabular}

TABLE 2: Saturation test images result.

Reference images $K_{1}=1.5 \quad K_{2}=2.0$

To keep the consistency of image color, we need $a_{1}^{*}$ and $a_{2}^{*}$ to have same sign symbol.

(7) According to (5), we have $Y_{2}=Y_{1}$ so that we can get $f\left(Y_{2} / Y_{0}\right)$ from the

$$
f(I)= \begin{cases}I^{1 / 3}, & \text { for } I>0.008856 \\ 7.787 I+\frac{16}{116}, & \text { otherwise. }\end{cases}
$$

(8) According to the CIEDE2000, $f\left(X_{2} / X_{0}\right), f\left(Z_{2} / Z_{0}\right)$ are given by $f\left(X_{2} / X_{0}\right)=\left(a_{2}^{*} / 500\right)+f\left(Y_{2} / Y_{0}\right)$ and $f\left(Z_{2} / Z_{0}\right)=f\left(Y_{2} / Y_{0}\right)-\left(b_{2}^{*} / 200\right)$. Put $f\left(X_{2} / X_{0}\right), f\left(Z_{2} / Z_{0}\right)$ into

$$
I= \begin{cases}f(I)^{3}, & \text { if } f(I)>0.2069 \\ \frac{f(I)-16 / 116}{7.787}, & \text { otherwise }\end{cases}
$$

we can get $X_{2}, Z_{2}$, where $I=X_{i} / X_{0}, Y_{i} / Y_{0}$, and $Z_{i} / Z_{0}$.
(9) The $R_{2}, G_{2}$, and $B_{2}$ of saturation test image are given by

$$
\left(\begin{array}{l}
R_{2} \\
G_{2} \\
B_{2}
\end{array}\right)=A^{-1}\left(\begin{array}{l}
X_{2} \\
Y_{2} \\
Z_{2}
\end{array}\right)
$$

the matrix $A$ is the same as in step (3).

\section{Experimental Systems and AssessmentResults}

The experimental environment was set according to the basic conditions for observation room. Our experimental monitor is founder FN980-WT, which has a resolution of $1440 \times$ 900 32-bit true color. To get repeatable experimental results, we used light source $D_{65}$ and calibrated the color temperature as $6500 \mathrm{~K}$, brightness as 80 , contrast as 70, and observable color grade as 61 .

Figure 1 shows a series of saturation test ladder images with equal difference. The $R, G$, and $B$ of the left most image 
are 120,150 , and 120 as the reference image. The subjective saturation coefficient $K_{n}$ is incremented from 1 to 7 . Table 1 shows the detailed experimental calculating parameters.

In addition, we have designed a set of saturation test images based on the LIVE database [6] as the reference images. LIVE database contains 29 different high resolution reference images. In Table 2, we listed two reference images randomly chosen from LIVE database and their corresponding saturation test images with saturation coefficient $K_{n}$ as $1.5,2.0$, and 2.5. For each group of reference and saturation test images, we can find by our subjective perception that the saturation of these images with same lightness and hue increased linearly with the subjective saturation coefficient $K_{n}$ as $C_{n}^{\prime}=K_{n} * C_{0}^{\prime}$. This experiment proves that our method can generate a series of saturation test images consistent with subjective perception.

\section{Conclusion}

In summary, we have proposed a saturation test image design method by exploiting the saturation parameter $C^{\prime}$ in CIEDE2000 color difference formula. Using this method, we can generate a series of the so-called saturation test images with different saturation but same lightness and hue. It is found experimentally that subjective visual perceptional is nice consistent with the saturation test images. And the vision perception is linear with the saturation parameters $\mathrm{C}^{\prime}$ of test images. Various applications can be found for this kind of saturation test images, for example, in the checking of color masking effect in visual experiments and the testing of the visual effects of image similarity component. Similar design idea can be also exploited to design other test images such as hue or lightness test images.

\section{Acknowledgments}

This work was supported by Fundamental Research Funds for the Central Universities (no. WK2100230002), National Science and Technology Major Project (no. 2010ZX03004003), National Natural Science Foundation of China (no. 60872162), and Young Research Foundation of Anhui University (no. KJQN1012).

\section{References}

[1] A. C. Bovik, "Perceptual video processing: seeing the future," Proceedings of the IEEE, vol. 98, no. 11, pp. 1799-1803, 2010.

[2] A. K. Moorthy and A. C. Bovik, "Visual quality assessment algorithms: what does the future hold?" Multimedia Tools and Applications, vol. 51, no. 2, pp. 675-696, 2011.

[3] A. C. Bovik, "What you see is what you learn," IEEE Signal Processing Magazine, vol. 27, no. 5, pp. 117-123, 2010.

[4] C. Yim and A. C. Bovik, "Quality assessment of deblocked images," IEEE Transactions on Image Processing, vol. 20, no. 1, pp. 88-98, 2011.

[5] L. Zhang, L. Zhang, X. Mou, and D. Zhang, "FSIM: a feature similarity index for image quality assessment," IEEE Transactions on Image Processing, vol. 20, no. 8, pp. 2378-2386, 2011.
[6] H. R. Sheikh, Z. Wang, L. Cormack, and A. C. Bovik, "LIVE Image Quality Assessment Database Release 2," http:// live.ece.utexas.edu/research/quality.

[7] VQEG, "Final report from the video quality experts group on the validation of objective models of video quality assessment," http://www.its.bldrdoc.gov/vqeg/.

[8] Tampere Image Database 2008 TID2008, version 1.0, http:// www.ponomarenko.info/tid2008.htm.

[9] J. Ming, H. C. Liu, and Y. Yang, "A spatial extension method of Weber-Fechner Law," in Proceedings of the 2nd International Conference on Information Science and Engineering (ICISE'10), pp. 4316-4319, December 2010.

[10] M. R. Luo, G. Cui, and B. Rigg, "The development of the CIE 2000 colour-difference formula: CIEDE2000," Color Research and Application, vol. 26, no. 5, pp. 340-350, 2001.

[11] R. G. Kuehni, “CIEDE2000, Milestone or final answer?” Color Research and Application, vol. 27, no. 2, pp. 126-127, 2002.

[12] M. R. Luo, G. Cui, and B. Rigg, "Further comments on CIEDE2000," Color Research and Application, vol. 27, no. 2, pp. 127-128, 2002.

[13] S. Yu and F. Guo, Principles of Television, China National Defence Industry Press, 1994. 

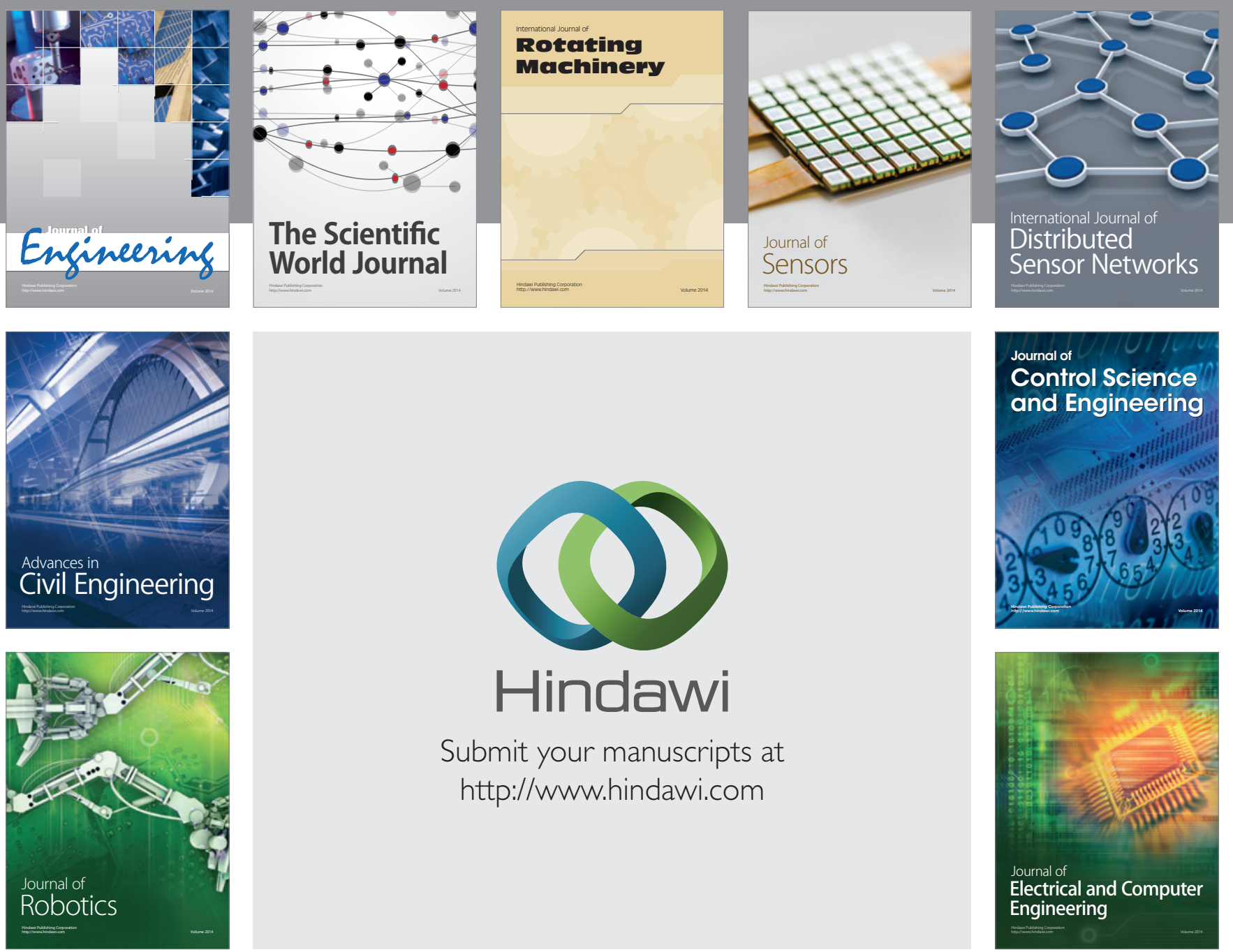

Submit your manuscripts at

http://www.hindawi.com
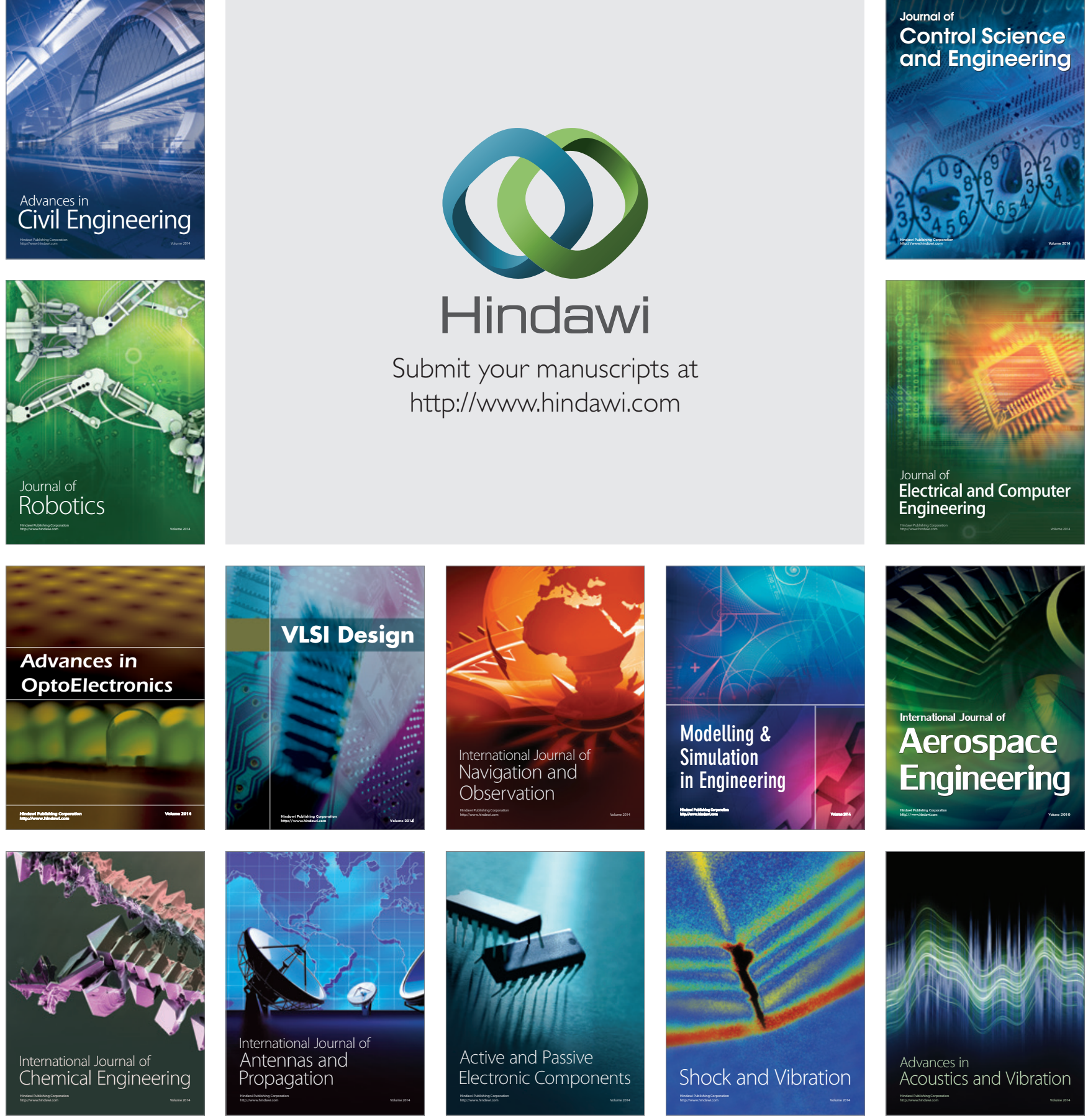